\title{
SELF-COMPLEMENTARY VERTEX-TRANSITIVE GRAPHS OF ORDER A PRODUCT OF TWO PRIMES
}

\author{
CAI HENG LI and GUANG RAO ${ }^{凶}$
}

(Received 26 March 2013; accepted 2 April 2013; first published online 13 June 2013)

\begin{abstract}
In this short paper, we characterise graphs of order $p q$ with $p, q$ prime which are self-complementary and vertex-transitive.
\end{abstract}

2010 Mathematics subject classification: primary 05C25; secondary 05E18.

Keywords and phrases: self-complementary, vertex-transitive.

\section{Introduction}

Throughout this paper, all graphs are simple. Let $\Gamma=(V, E)$ be a graph with vertex set $V$ and edge set $E$. The complement $\bar{\Gamma}$ of $\Gamma$ is the graph with the same vertex set $V$ such that for any two distinct vertices $u, v \in V,\{u, v\}$ is an edge of $\bar{\Gamma}$ if and only if $\{u, v\}$ is not an edge of $\Gamma$. A graph $\Gamma$ is said to be self-complementary if $\Gamma$ is isomorphic to $\bar{\Gamma}$.

A permutation of $V$ is called an automorphism of the graph $\Gamma=(V, E)$ if it preserves the edge set $E$. The automorphism group of $\Gamma$ consists of all automorphisms of $\Gamma$, denoted by Aut $\Gamma$. A graph $\Gamma=(V, E)$ is called vertex-transitive if Aut $\Gamma$ is transitive on the vertex set $V$.

The graphs that are both self-complementary and vertex-transitive are called selfcomplementary vertex-transitive (SCVT) graphs. It is generally still hard to study the general SCVT graphs, thus the study of small families of SCVT graphs becomes interesting, and also provides good examples for reference. SCVT graphs of prime order are well understood; see for example [1]. Thus, it is natural to study the next unknown case, the graphs of order a product of two primes. Some of the basic properties of this family of graphs have been established in the literature. In 1979, Zelinka [20] conjectured that if there is an SCVT graph of order $p q$ with $p, q$ distinct primes then both $p, q$ are congruent to 1 modulo 4 . This conjecture was verified in [7]. However, the structural description of these graphs was left open.

In this paper, we study SCVT graphs of order $p q$, with $p, q$ being primes, not necessarily distinct. Applying the methods developed in [5, 10], we successfully classify all these graphs.

(C) 2013 Australian Mathematical Publishing Association Inc. 0004-9727/2013 \$16.00 
Theorem 1.1. Let $\Gamma$ be an SCVT graph of order a product of two primes. Then $\Gamma$ is one of the following:

(i) a lexicographic product of two SCVT graphs;

(ii) a normal Cayley graph of an abelian group.

With this result in mind, we have a simple characterisation for the case where two primes are distinct.

Corollary 1.2. The SCVT graphs of order a product of two distinct primes are circulants.

We make some remarks on Theorem 1.1. By the characterisation of Theorem 1.1, we can construct all such graphs: if $\Gamma$ is a lexicographic product, the SCVT graphs of prime order are all well characterised (see [1]); if $\Gamma$ is a normal Cayley graph, it can be easily constructed by the definite group (see Section 2).

The study of SCVT graphs has a long history; see the surveys of Beezer [2] and the first author [8]. The following is a very brief review.

A graph $\Gamma=(V, E)$ is called a circulant if Aut $\Gamma$ contains a cyclic subgroup which is transitive on the vertices. Circulants are vertex-transitive graphs. The first family of SCVT graphs was constructed as circulants by Sachs [16], and later Zelinka [20], Mathon [12], Rao [15], Suprunenko [17], Liskovets and Poschel [11] and Jajcay and Li [6] also studied self-complementary circulants.

In the 1990s, the orders of SCVT graphs were completely determined. Alspach et al. [1] determined the orders of self-complementary circulants, and later Fronček et al. [3] gave a different proof. Finally, in 1999, Muzychuck [13] completely determined the orders of general SCVT graphs by proving that the Sylow subgraphs are also self-complementary.

The class of edge-transitive self-complementary graphs was classified by Zhang [21] and Peisert [14]. Most known SCVT graphs are constructed as Cayley graphs (defined later). The first family of SCVT graphs which are not Cayley graphs was obtained by Li and Praeger [10] in 2003. Since 2000, the study of SCVT graphs has been significantly progressed by Li and Praeger's work [10] and their joint work with Guralnick et al. [5] for the vertex-primitive case. Our main theorem in this paper heavily depends on these results.

\section{Constructions}

Let $\Gamma=(V, E)$ be a self-complementary graph. An isomorphism $\sigma$ between $\Gamma$ and $\bar{\Gamma}$ is called a complementing isomorphism. Since $\sigma$ always interchanges $\Gamma$ and $\bar{\Gamma}$, replacing $\sigma$ by an odd power of $\sigma$, we may assume that $\sigma$ is of 2-power order. Moreover, $\sigma$ does not fix any pair of vertices, so $\sigma$ has order divisible by 4 . Furthermore, if $\Gamma$ is vertex-transitive, we may assume that $\sigma$ fixes a vertex $v$. Then $\sigma^{2}$ only fixes $v$, for otherwise $\sigma$ would fix a pair of vertices, which is not possible.

LEMMA 2.1. A complementing isomorphism of a self-complementary graph has order divisible by 4 . 
In Theorem 1.1, SCVT graphs of order a product of two primes are divided into two classes. One of them is involved in the lexicographic product, defined as follows.

Let $\Gamma_{1}=\left(V_{1}, E_{1}\right)$ and $\Gamma_{2}=\left(V_{2}, E_{2}\right)$ be graphs. Then the lexicographic product of $\Gamma_{1}$ and $\Gamma_{2}$ is the graph with vertex set $V_{1} \times V_{2}$ such that two vertices $\left(u_{1}, u_{2}\right)$ and $\left(v_{1}, v_{2}\right)$ are adjacent if and only if either $\left\{u_{1}, v_{1}\right\} \in E_{1}$, or $u_{1}=v_{1}$ and $\left\{u_{2}, v_{2}\right\} \in E_{2}$. This graph is denoted by $\Gamma_{1}\left[\Gamma_{2}\right]$. The lexicographic product provides a method for constructing SCVT graphs based on the following properties (see [2]).

LeMma 2.2. If both $\Gamma_{1}$ and $\Gamma_{2}$ are self-complementary (vertex-transitive), then so is $\Gamma_{1}\left[\Gamma_{2}\right]$.

The second class of graphs in Theorem 1.1 is involved with the normal Cayley graphs.

For a finite group $R$, let $R^{\#}=R \backslash\{1\}$, the set of all nonidentity elements of $R$. For a subset $S \subseteq R^{\#}$ with $S=S^{-1}:=\left\{s^{-1} \mid s \in S\right\}$, the associated Cayley graph Cay $(R, S)$ is the graph $\Gamma=(V, E)$ with $V=R$ such that two vertices $a, b \in R$ are adjacent if and only if $b a^{-1} \in S$. Clearly, the complement of Cay $(R, S)$ is the graph Cay $\left(R, R^{\#} \backslash S\right)$.

For an element $g \in R$, let

$$
\hat{g}: x \mapsto x g, \quad \text { for all } x \in R,
$$

the right multiplication of the element $g \in R$ on the elements of $R$. Let $\hat{R}=\{\hat{g} \mid g \in R\}$. Then $\hat{R}$ is an automorphism subgroup of Cay $(R, S)$, acting regularly on $V$. Thus, Cayley graphs are vertex-transitive. Conversely, for a graph $\Gamma$, if Aut $\Gamma$ contains a subgroup which is regular on the vertex set and isomorphic to a group $R$, then $\Gamma$ is a Cayley graph of $R$. In particular, a circulant is a Cayley graph of a cyclic group. Further, we have the following simple lemma, which was proved in [4, Lemma 2.1].

Lemma 2.3. Let $\Gamma=$ Cay $(R, S)$. Then

$$
\mathbf{N}_{\text {Aut } \Gamma}(\hat{R})=\hat{R}: \text { Aut }(R, S),
$$

where Aut $(R, S)=\left\{\sigma \in\right.$ Aut $\left.(R) \mid S^{\sigma}=S\right\}$.

In the case where $\hat{R}$ is normal in Aut $\Gamma$, then $\Gamma=$ Cay $(R, S)$ is called a normal Cayley graph of $R$; refer to [19]. Lemma 2.3 tells us that every automorphism of $R$ which fixes the subset $S$ (setwise) is an automorphism of the Cayley graph. On the other hand, an automorphism of $R$ which does not fix $S$ induces an isomorphism between Cayley graphs, namely, any automorphism $\sigma \in$ Aut $(R)$ induces an isomorphism from Cay $(R, S)$ to Cay $\left(R, S^{\sigma}\right)$. Therefore, if a Cayley graph $\Gamma=$ Cay $(R, S)$ satisfies

$$
S^{\sigma}=R^{\#} \backslash S, \quad \text { for some } \sigma \in \text { Aut }(R),
$$

then $\Gamma$ is self-complementary. In this case, the automorphism $\sigma$ is a complementing isomorphism between $\Gamma$ and $\bar{\Gamma}$. As we discussed before, we may assume that $\sigma$ fixes the vertex that corresponds to the identity of $R$. Moreover, this is the only 
vertex which $\sigma^{2}$ fixes, that is, $\sigma^{2}$ is a fixed-point-free automorphism of $R$. This observation leads to a generic construction for self-complementary Cayley graphs; see [10, Construction 3.1].

Let $R$ be an abelian group of order $p q$ with $p, q$ prime. Then $R \cong \mathbb{Z}_{p}^{2}$ or $\mathbb{Z}_{p q}$. The next lemma tells us when $R$ has an automorphism $\sigma$ of order a power of 2 such that $\sigma^{2}$ is fixed-point-free.

Lemma 2.4. Let $R$ be an abelian group of order $p q$ where $p, q$ are odd prime numbers. Then there exists $\sigma \in$ Aut $(R)$ such that $\sigma^{2}$ is of order a power of 2 and fixed-point-free if and only if $R \cong \mathbb{Z}_{p}^{2}$, or $R$ is cyclic with $p, q \equiv 1(\bmod 4)$.

Proof. First, assume that $R=\langle a\rangle \times\langle b\rangle \cong \mathbb{Z}_{p}^{2}$. Let $\sigma \in$ Aut $(R)$ be such that

$$
\sigma: a \rightarrow b, b \rightarrow a^{-1}
$$

Then $\sigma^{2}$ maps each element $x \in R$ to the inverse $x^{-1}$, hence $\sigma^{2}$ is fixed-point-free, and $\sigma^{2}$ has order two.

Next, assume that $R=\langle a\rangle=\mathbb{Z}_{p^{2}}$. Then Aut $(R)=\mathbb{Z}_{p(p-1)}$, and thus 4 divides the order of Aut $(R)$ if and only if 4 divides $p-1$. Moreover, if $\sigma$ is an automorphism of $R$ of order divisible by 4 , then $\sigma^{2}$ obviously fixes no nonidentity element of $R$.

Finally, assume that $p \neq q$, and $R=\langle a\rangle \times\langle b\rangle \cong \mathbb{Z}_{p} \times \mathbb{Z}_{q}$. Then Aut $(R)=$ Aut $(\langle a\rangle) \times$ Aut $(\langle b\rangle)=\langle x\rangle \times\langle y\rangle \cong \mathbb{Z}_{p-1} \times \mathbb{Z}_{q-1}$. If $p, q \equiv 1(\bmod 4)$, then let $x_{1} \in$ Aut $(\langle a\rangle), y_{1} \in$ Aut $(\langle b\rangle)$ be of order four. Then $\left(x_{1}, y_{1}\right) \in$ Aut $(R)$ is of order four and the square $\left(x_{1}, y_{1}\right)^{2}$ fixes no nonidentity element of $R$. Conversely, suppose that $p \not \equiv 1(\bmod 4)$. Let $\sigma \in$ Aut $(R)$ be of order a power of 2. Then $\sigma=\left(x^{i}, y^{j}\right)$ such that $x^{i}$ is of order two, and $\sigma^{2}=\left(x^{2 i}, y^{2 j}\right)=\left(1, \tau^{2 j}\right)$. Clearly, $\sigma^{2}$ fixes the element $a$.

For a group $R$ of order $p q$ and an automorphism $\sigma$ with property (*), we can easily construct self-complementary Cayley graphs of $R$ as below (refer to [8]).

Construction 2.5. Let $R$ be a group, and let $\sigma \in$ Aut $(R)$ be such that $\sigma^{2}$ is fixed-pointfree and of 2-power order, and $g^{-1} \in g^{\left\langle\sigma^{2}\right\rangle}$ for all $g \in R$. Let $\Gamma=$ Cay $(R, S)$ be a Cayley graph with $S$ defined as follows:

(1) Let $\Delta_{1}, \Delta_{2}, \ldots, \Delta_{r}$ be the $\langle\sigma\rangle$-orbits on $R^{\#}$, and label the two orbits of $\left\langle\sigma^{2}\right\rangle$ on $\Delta_{i}$ as $\Delta_{i}^{+}$and $\Delta_{i}^{-}$, where $1 \leq i \leq r$.

(2) Set $S=\bigcup_{i=1}^{r} \Delta_{i}^{\varepsilon_{i}}$ where $\varepsilon_{i}=+$ or - . (We remark that there are $2^{r}$ different choices for such a subset $S$.)

Lemma 2.6 [10, Lemma 3.2]. Each graph $\Gamma$ constructed in Construction 2.5 is selfcomplementary.

This construction method was generated in [9] with regard to the coset graphs.

Definition 2.7. Let $G$ be a group, and let $H$ be a core-free subgroup of $G$, and $S \subset G \backslash H$. Define the coset graph $\Gamma=\operatorname{Cos}(G, H, H S H)$ to be the digraph with vertex set $[G: H]$ such that $H x$ and $H y$ are adjacent if and only if $y x^{-1} \in H S H$. 
The following theorem provides a generic construction method for SCVT graphs.

Theorem 2.8 (Li and Praeger [9]). Let $G$ be a finite group, and let $H$ be a corefree subgroup of $G$. Then there exists an undirected coset graph of $G$ with respect to $H$ which is self-complementary if and only if there exists an automorphism $\sigma \in$ Aut $(G)$ of order a power of 2 such that $H^{\sigma}=H$, and for each $g \in G \backslash H,(H g H)^{\sigma} \notin$ $\left\{\mathrm{HgH}, \mathrm{Hg}^{-1} \mathrm{H}\right\}$.

\section{Proof of the main theorem}

Let $\Gamma=(V, E)$ be a self-complementary graph of order $p q$ with $p, q$ prime. Let $G=$ Aut $\Gamma$, let $\sigma$ be a complementing isomorphism between $\Gamma$ and $\bar{\Gamma}$, and let $X=\langle G, \sigma\rangle$. Then $\sigma^{2} \in G, X=G . \mathbb{Z}_{2}$. We choose $\sigma$ such that $o(\sigma)=2^{f}, f \geq 2$. Since $G$ is vertextransitive, without loss of generality, we assume that $\sigma$ fixes a vertex $v \in V$, and so $X_{v}=\left\langle G_{v}, \sigma\right\rangle$.

3.1. Primitive case We first consider the vertex-primitive case.

Lemma 3.1. Assume that $X$ is primitive on the vertex set $V$. Then $p=q$, and $X$ is affine of degree $p^{2}$. Moreover, the vertex stabiliser $X_{v}$ satisfies one of the following:

(i) $\quad X_{v} \leq \mathrm{GL}(1, p)<\mathrm{S}_{2}$, and $X_{v} \leq \mathrm{GL}(1, p) \times \mathrm{GL}(1, p)$;

(ii) $X_{v} \leq \Gamma \mathrm{L}\left(1, p^{2}\right)$, and $\left(\left|X_{v}\right|, p+1\right)>2$;

(iii) $\mathrm{Q}_{8} \leq X_{v} \leq \mathbb{Z}_{p-1} \circ \mathrm{GL}(2,3)$;

(iv) $\operatorname{SL}(2,5)<X_{v} \leq \mathbb{Z}_{p-1} \circ \mathrm{SL}(2,5)$, where $p \equiv \pm 1(\bmod 10)$.

Proof. By [5, Theorem 1.3 and Corollary 1.4], the group $X$ is affine, or in product action with $|V|=\left(\frac{1}{2} r^{2}\left(r^{2}+1\right)\right)^{\ell}$, where $r$ is a power of an odd prime, and $\ell \geq 2$. In the latter case, $|V|$ is not a product of two primes, so $X$ is affine of degree $|V|=p^{2}$, and $p=q$.

Thus, $X=\mathbb{Z}_{p}^{2}: X_{v}$ where $X_{v} \leq \mathrm{GL}(2, p)$ is irreducible on the vector space $\operatorname{GF}(p)^{2}$. In particular, $\sigma^{2}$ is a fixed-point-free automorphism of $\mathbb{Z}_{p}^{2}$. We first point out that $p \neq 2$ as $\operatorname{GL}(2,2)$ does not contain an element of order four. Since $G$ is transitive on $V$, $\left|X_{v}: G_{v}\right|=|X: G|=2$. Let $M$ be a maximal subgroup of $\mathrm{GL}(2, p)$ containing $X_{v}$. Then $M$ is one of the following (see [18, page 417]):

$$
\mathrm{GL}(1, p) \imath \mathrm{S}_{2} ; \Gamma \mathrm{L}\left(1, p^{2}\right) ; \mathbb{Z}_{p-1} \circ \mathrm{GL}(2,3) ; \mathbb{Z}_{p-1} \circ \mathrm{SL}(2,5) \text { with } p \equiv \pm 1(\bmod 10) .
$$

If $M=\mathrm{GL}(1, p)$ 乙 $\mathrm{S}_{2}$, since $X_{v}$ is irreducible, $X_{v} \nless \mathrm{GL}(1, p) \times \mathrm{GL}(1, p)$, as in part (i). For the case $M=\Gamma \mathrm{L}\left(1, p^{2}\right)$, if $\left(\left|X_{v}\right|, p+1\right) \leq 2$, then $X_{v}$ is conjugate to a subgroup of $\operatorname{GL}(1, p) \succ S_{2}$, and so part (i) or (ii) is satisfied. For the case $M=\mathbb{Z}_{p-1} \circ \mathrm{GL}(2,3)$, if $X_{v}$ does not contain $\mathrm{Q}_{8}$, then part (i) or (ii) is satisfied, and if $X_{v} \geq \mathrm{Q}_{8}$, then part (iii) is satisfied. Finally, for $M=\mathbb{Z}_{p-1} \circ \operatorname{SL}(2,5)$, we may assume that $X_{v}$ is not a subgroup of $\mathrm{GL}(1, p) \succ \mathrm{S}_{2}, \Gamma \mathrm{L}\left(1, p^{2}\right)$ and $\mathbb{Z}_{p-1} \circ \mathrm{GL}(2,3)$. Thus, $X_{v}$ is insoluble and contains $\operatorname{SL}(2,5)$, and as $X_{v}$ has a subgroup $G_{v}$ of index 2, we further have $X_{v}>\operatorname{SL}(2,5)$, as in part (iv). 
3.2. Imprimitive case In this subsection, we consider the case where $X$ is imprimitive on $V$. Let $\mathcal{B}$ be a nontrivial $X$-invariant partition of $V$, called a block system of the action of $X$ on $V$. Each element $B \in \mathcal{B}$ is called a block. Without loss of generality, we assume that $|\mathcal{B}|=q$. Then each block has size $p$. Denote by $[B]_{\Gamma}$ the induced subgraph of $\Gamma$ on $B$, namely, the graph whose vertex set is $B$ and whose edge set consists of all edges of $\Gamma$ which lie inside $B$. The quotient graph $\Gamma_{\mathcal{B}}$ of $\Gamma$ is the graph with vertex set $\mathcal{B}$ such that two vertices $B, C \in \mathcal{B}$ are adjacent if and only if some $u \in B$ and some $v \in C$ are adjacent in $\Gamma$.

To treat the imprimitive case, we need a result of Li and Praeger [10].

Theorem 3.2. Assume that $X$ is imprimitive on $V$. Then:

(i) the induced subgraph $[B]_{\Gamma}$ is self-complementary, $G_{B}^{B} \leq$ Aut $[B]_{\Gamma}$, and $\sigma$ induces a complementing isomorphism between $[B]_{\Gamma}$ and $\overline{[B]_{\Gamma}}$;

(ii) there is a self-complementary graph $\Sigma$ with vertex set $\mathcal{B}$ such that $G^{\mathcal{B}} \leq$ Aut $\Sigma$ and each element of $X^{\mathcal{B}} \backslash G^{\mathcal{B}}$ is a complementing isomorphism between $\Sigma$ and $\bar{\Sigma}$.

Lemma 3.3. Using the notation defined above, we have $\mathbb{Z}_{p} \triangleleft X_{B}^{B} \leq \operatorname{AGL}(1, p)$, and $\mathbb{Z}_{q} \triangleleft X^{\mathcal{B}} \leq \operatorname{AGL}(1, q)$.

Proof. By Theorem 3.2(i) the induced subgraph $[B]_{\Gamma}$ on $B$ is self-complementary. Thus, $G_{B}^{B}$ is not 2-transitive, and neither is $X_{B}^{B}$. Since $|B|=p$, it follows that $\mathbb{Z}_{p} \triangleleft X_{B}^{B} \leq$ $\operatorname{AGL}(1, p)$.

Similarly, by Theorem 3.2(ii), $G^{\mathcal{B}}$ is not 2-transitive, and neither is $X^{\mathcal{B}}$. Hence we conclude that $\mathbb{Z}_{q} \triangleleft X^{\mathcal{B}} \leq \operatorname{AGL}(1, q)$.

Lemma 3.4. The action of $X$ on $\mathcal{B}$ is unfaithful.

Proof. Suppose that $X \cong X^{\mathcal{B}}$ is faithful. By Lemma 3.3, we have $X \cong X^{\mathcal{B}} \leq$ $\operatorname{AGL}(1, q) \cong \mathbb{Z}_{q}: \mathbb{Z}_{q-1}$, and all the $q^{\prime}$-subgroups of $X$ are cyclic. Note that $G_{v}$ is core-free in $G$, so by Theorem 2.8 it follows that $\sigma$ fixes no double cosets $G_{v} g G_{v}$, that is, $G_{v} g^{\sigma} G_{v} \neq G_{v} g G_{v}$ for each $g \in G \backslash G_{v}$. Since $\left|X: X_{v}\right|=p q$, it follows that $G_{v}<X_{v}$ is properly contained in a Hall $q^{\prime}$-subgroup $X_{q^{\prime}}$, that is, $G_{v}<X_{q^{\prime}} \leq \mathbb{Z}_{q-1}$. Note that $\sigma \in X_{v}<X_{q^{\prime}}$, so $\sigma$ centralises all elements in $X_{q^{\prime}}$. In particular, for any $h \in\left(X_{q^{\prime}} \cap G\right) \backslash G_{v}$, we have $G_{v} h^{\sigma} G_{v}=G_{v} h G_{v}$, which is not possible. Therefore, $X$ is not faithful on $\mathcal{B}$.

Let $K=X_{(\mathcal{B})}$ be the kernel of $X$ acting on $\mathcal{B}$. Then $1 \neq K \triangleleft X$ by Lemma 3.4, and $1 \neq K^{B} \triangleleft X_{B}^{B}$ for some $B \in \mathcal{B}$. Thus, $K^{B}=\mathbb{Z}_{p}: \mathbb{Z}_{r}$ for some $r$ dividing $p-1$. For two blocks $B, C \in \mathcal{B}$, we denote by $[B, C]$ the subgraph of $\Gamma$ with vertex set $B \cup C$ and edge set consisting of all the edges of $\Gamma$ between $B$ and $C$.

Lemma 3.5. If $p^{2}|| K \mid$, then $\Gamma=\Gamma_{1}\left[\Gamma_{2}\right]$, where $\Gamma_{1}$ and $\Gamma_{2}$ are self-complementary circulants of order $q$ and $p$, respectively.

Proof. Let $B_{1}=B$ and let $B_{2} \in \mathcal{B}$ be distinct from $B$. Consider the subgraph $\left[B_{1}, B_{2}\right]$. Since $X$ is transitive on the $q$ blocks, relabelling if necessary, we assume that $\mathcal{B}=\left\{B_{1}, B_{2}, \ldots, B_{q}\right\}$ such that $\left[B_{i}, B_{i+1}\right] \cong\left[B_{1}, B_{2}\right]$ for all $i \in\{1, \ldots, q-1\}$. 
Let $P$ be a Sylow $p$-subgroup of $K$, and let $P_{(B)}$ be the kernel of $P$ acting on $B$. Then $P / P_{(B)} \cong P^{B} \cong \mathbb{Z}_{p}$, and $P_{(B)} \neq 1$. Hence $P_{(B)}$ acts nontrivially on some $B_{k}$ with $k \neq 1$. Without loss of generality, assume that $k$ is the smallest integer with this property. Then $P_{(B)} \leq P_{\left(B_{k-1}\right)}$ is transitive on $B_{k}$ as $\left|B_{k}\right|=p$. Notice that $K$ is transitive on $B_{k-1}$. If $\left[B_{1}, B_{2}\right]$ is nonempty, then so is $\left[B_{k-1}, B_{k}\right]$, and we have $\left[B_{1}, B_{2}\right] \cong\left[B_{k-1}, B_{k}\right]=\mathbf{K}_{p, p}$. Therefore, for any two blocks $B, C \in \mathcal{B}$, either $[B, C]$ is empty, or $[B, C]=\mathbf{K}_{p, p}$. Let $E^{\prime}=E \backslash\left(\bigcup_{i=1}^{q} B_{i} \times B_{i}\right)$. Then $\Gamma^{\prime}=\left(V, E^{\prime}\right) \cong \Gamma_{\mathcal{B}}\left[\overline{\mathbf{K}_{p}}\right]$. Since $\sigma$ preserves the block system $\mathcal{B}, \sigma$ maps $\Gamma_{\mathcal{B}}\left[\overline{\mathbf{K}_{p}}\right]$ to $\overline{\Gamma_{\mathcal{B}}}\left[\overline{\mathbf{K}_{p}}\right]$, and so $\Gamma_{\mathcal{B}}$ is self-complementary.

Let $\Gamma_{1}=\Gamma_{\mathcal{B}}$ and $\Gamma_{2}=[B]_{\Gamma}$. Then $\Gamma=\Gamma_{1}\left[\Gamma_{2}\right]$. By Theorem 3.2 the induced subgraph $[B]_{\Gamma}=\Gamma_{2}$ is also self-complementary. Thus the proof is complete.

Lemma 3.6. Assume that $p^{2}$ does not divide $|K|$. Then $X=R: X_{v}$, where $R$ is abelian of order $p q$, and regular on $V$.

Proof. Suppose that $1 \neq K_{(B)} \triangleleft K$. Then there exists $B^{\prime} \in \mathcal{B}$ such that $K_{(B)}$ acts on $B^{\prime}$ transitively, and hence $p|| K_{(B)} \mid$. This yields that $p^{2}$ divides $|K|$, which is a contradiction. So $K \cong K^{B}=\mathbb{Z}_{p}: \mathbb{Z}_{r}$ for some $r$ dividing $p-1$. Now $X^{\mathcal{B}}=\mathbb{Z}_{q}: \mathbb{Z}_{s}$ with $s \mid(q-1)$ and $s \neq 1$. We consider the extension

$$
X=K \cdot X^{\mathcal{B}}=\left(\mathbb{Z}_{p}: \mathbb{Z}_{r}\right) \cdot\left(\mathbb{Z}_{q}: \mathbb{Z}_{s}\right) .
$$

Let $P$ be a Sylow $p$-subgroup of $K$. Then $P \cong \mathbb{Z}_{p}$ is normal in $X$, and Aut $(P)=\mathbb{Z}_{p-1}$ is abelian. Thus, $X / \mathbf{C}_{X}(P) \leq$ Aut $(P)$ is abelian, and so $\mathbf{C}_{X}(P) \geq P . Q$, where $Q$ is the unique Sylow $q$-subgroup of $X^{\mathcal{B}}$. Since $|P . Q|=p q$, either $P . Q \cong \mathbb{Z}_{p q}$ is cyclic, or $p=q$ and $P . Q \cong \mathbb{Z}_{p}^{2}$. Letting $R=P . Q$, we have that $R$ is abelian, and regular on $V$. Therefore, $X=R: X_{v}$.

3.3. Proofs of the main results The proof of Theorem 1.1 can now be given briefly as follows.

Proof of Theorem 1.1. Let $\Gamma=(V, E)$ be an SCVT graph of order $p q$ with $p, q$ prime. Let $G=$ Aut $\Gamma$, and let $\sigma$ be a complementing isomorphism between $\Gamma$ and $\bar{\Gamma}$. Let $X=\langle G, \sigma\rangle$.

If $X$ is primitive on the vertex set $V$, then by Lemma 3.1, $\Gamma$ is a normal Cayley graph of $\mathbb{Z}_{p}^{2}$.

If $X$ is imprimitive on $V$, then, by Lemmas 3.5 and 3.6, Theorem 1.1 holds.

The proof of Corollary 1.2 follows from the lemma below.

LEMMA 3.7. If $p \neq q$, then either:

(i) $\quad \Gamma=\Gamma_{1}\left[\Gamma_{2}\right]$, where $\Gamma_{1}$ and $\Gamma_{2}$ are self-complementary circulants of order $q$ and $p$, respectively; or

(ii) Aut $\Gamma=\mathbb{Z}_{p q}: H$, where $H<\mathbb{Z}_{p-1} \times \mathbb{Z}_{q-1}$.

In particular, $\Gamma$ is a circulant. 
Proof. Since $p \neq q$, it follows that $X$ is imprimitive by Lemma 3.1. By Lemmas 3.5 and 3.6, either part (i) or part (ii) is satisfied. If $\Gamma$ satisfies part (ii), then Aut $\Gamma=\mathbb{Z}_{p q}$ : $H$, and it follows from Lemma 2.3 that $H<$ Aut $\left(\mathbb{Z}_{p q}\right)=\mathbb{Z}_{p-1} \times \mathbb{Z}_{q-1}$.

Assume that $\Gamma=\Gamma_{1}\left[\Gamma_{2}\right]$. Then Aut $\Gamma_{2}$ 乙 Aut $\Gamma_{1} \leq$ Aut $\Gamma$. Let $x \in$ Aut $\Gamma_{2}, y \in$ Aut $\Gamma_{1}$ be of order $p$ and $q$, respectively. Note that $\langle y\rangle$ cyclically permutes the $q$ entries of the base group, so $z=(x, 1, \ldots, 1) y$ has order $p q$, and $\langle z\rangle$ is regular on the vertex set of $\Gamma$. Thus, $\Gamma$ is a circulant.

\section{References}

[1] B. Alspach, J. Morris and V. Vilfred, 'Self-complementary circulant graphs', Ars Combin. 53 (1999), 187-191.

[2] R. A. Beezer, 'Sylow subgraphs in self-complementary vertex transitive graphs', Expo. Math. 24(2) (2006), 185-194.

[3] D. Fronček, A. Rosa and J. Širáň, 'The existence of selfcomplementary circulant graphs', European J. Combin. 17 (1996), 625-628.

[4] C. D. Godsil, 'On Cayley graphs isomorphisms', Ars Combin. 15 (1983), 231-246.

[5] R. M. Guralnick, C. H. Li, C. E. Praeger and J. Saxl, 'On orbital partitions and exceptionality of primitive permutation groups', Trans. Amer. Math. Soc. 356 (2004), 4857-4872.

[6] R. Jajcay and C. H. Li, 'Constructions of self-complementary circulants with no multiplicative isomorphisms', European J. Combin. 22(8) (2001), 1093-1100.

[7] C. H. Li, 'On self-complementary vertex-transitive graphs', Comm. Algebra 25 (1997), 39033908.

[8] C. H. Li, 'On finite graphs that are self-complementary and vertex-transitive', Australas. $J$. Combin. 18 (1998), 147-155.

[9] C. H. Li and C. E. Praeger, 'Self-complementary vertex-transitive graphs need not be Cayley graphs', Bull. Lond. Math. Soc. 33(6) (2001), 653-661.

[10] C. H. Li and C. E. Praeger, 'On partitioning the orbitals of a transitive permutation group', Trans. Amer. Math. Soc. 355 (2003), 637-653.

[11] V. Liskovets and R. Poschel, 'Non-Cayley-isomorphic self-complementary circulant graphs', J. Graph Theory 34 (2000), 128-141.

[12] R. Mathon, 'On selfcomplementary strongly regular graphs', Disc. Math. 69 (1988), 263-281.

[13] M. Muzychuk, 'On Sylow subgraphs of vertex-transitive self-complementary graphs', Bull. Lond. Math. Soc. 31(5) (1999), 531-533.

[14] W. Peisert, 'All self-complementary symmetric graphs', J. Algebra 240 (2001), 209-229.

[15] S. B. Rao, 'On regular and strongly regular selfcomplementary graphs', Disc. Math. 54 (1985), 73-82.

[16] H. Sachs, 'Über selbstcomplementäre Graphen', Publ. Math. Debrecen 9 (1962), 270-288.

[17] D. A. Suprunenko, 'Selfcomplementary graphs', Cybernetics 21 (1985), 559-567.

[18] M. Suzuki, Group Theory I (Springer, New York, 1986).

[19] M. Y. Xu, 'Automorphism groups and isomorphisms of Cayley digraphs', Discrete Math. 182(1-3) (1998), 309-319.

[20] B. Zelinka, 'Self-complementary vertex-transitive undirected graphs', Math. Slovaca 29 (1979), 91-95.

[21] H. Zhang, 'Self-complementary symmetric graphs', J. Graph Theory 16 (1992), 1-5. 
CAI HENG LI, School of Mathematics and Statistics,

The University of Western Australia, Crawley 6009, WA, Australia e-mail: cai.heng.li@uwa.edu.au

GUANG RAO, School of Mathematics and Statistics,

The University of Western Australia, Crawley 6009, WA, Australia e-mail: generalrao@hotmail.com 